\title{
Führen ohne Feinde: gewaltfreie Kommunikation im Pflegealltag
}

\author{
Al Weckert
}

(C) Springer-Verlag 2012

Dienstleister im Gesundheitswesen stehen unter enormen Kostendruck, gleichzeitig wird eine hochprofessionelle und fürsorgliche Arbeitshaltung erwartet. Daraus ergeben sich vier hauptsächliche Konfliktbereiche: zwischenmenschlicher Ärger im multiprofessionellen Team; Konflikte rund um Behandlungsfragen; strukturelle Konflikte (Personaleinsparungen, Umstrukturierungen etc.) in der Gesamtorganisation oder zwischen Teilbereichen; und Konfliktlinien im Umgang mit Patienten bzw. Kunden.

Interviews in Kliniken, Arztpraxen und bei Pflegedienstleistern ergeben umfangreichen Trainingsbedarf bei Führungskräften und Mitarbeitern. Diese wünschen sich eine zeitnahe Bearbeitung von Konflikten; einen geschützten Rahmen, um offen miteinander sprechen zu können; Handwerkszeug, um Bedürfnisse authentisch ausdrücken zu können; Einblick in das Gefühlsleben der Kollegen; gegenseitiges Vertrauen; eine verbesserte Kommunikation zwischen den Berufsgruppen; Transparenz und klarere Absprachen im multiprofessionellen Team; respektvollen Umgang zwischen unterschiedlichen Hierarchieebenen; Wertschätzung für die erbrachte Arbeitsleistung; Zeit und Handwerkszeug für Selbstreflexion und Selbstempathie; im Kontakt mit dem Patienten qualitativ mehr zu erfahren; auch in schwierigen Situationen eine wertschätzende Haltung bewahren können (auch bei „unsympathischen“ Kollegen und Patienten); Teamsitzungen, in denen jeder zu Wort kommt; Ansprechpartner „mit offenen Ohren“; offenes Aufeinander Eingehen, ohne an Anerkennung zu verlieren; und eine offenere Kommunikation über Sachfragen, um effizienter arbeiten zu können.

Anhand zahlreicher Beispiele aus Krankenhäusern und Pflegeeinrichtungen lässt sich zeigen, wie sich durch das

A. Weckert $(\triangle)$

EMPA|TRAIN Organisationsentwicklung, Berlin, Deutschland
Training von Gewaltfreier Kommunikation (nach Marshall Rosenberg) Konflikte effizient bearbeiten und eine wertschätzende Teamzusammenarbeit nachhaltig fördern lassen. Empathie in der Personalführung steigert die Arbeitszufriedenheit und senkt die Konfliktkosten (Krankenstände, Fluktuation, ,innere Kündigung“ usw.). Empathische Kommunikation verhilft Führungskräften zu einer wertschätzenden und auf gegenseitiger Unterstützung basierenden Verbindung zu ihren Mitarbeitern. Selbstempathie schützt Führungskräfte und Mitarbeiter vor Überforderung und Hilflosigkeit und bietet wirkungsvollen Schutz vor dem Burnout-Syndrom. 